\title{
HAMARTOMA HIPOTALAMICO ASOCIADO A DOS QUISTES ARACNOIDEOS. A PROPOSITO DE UN CASO
}

\section{Drs. Diana Martín $H^{(1)}$, Pablo Prieto $M^{(2)}$, Juan Carlos Paniagua $E^{(3)}$, Joaquin Benito $D^{(4)}$, Jesús Prieto $V^{(5)}$.}

1. Hospital Universitario de Salamanca. Servicio de Radiodiagnóstico. España

2. Hospital Universitario de Salamanca. Servicio de Endocrinología Pediátrica. España.

3. Hospital Universitario de Salamanca. Servicio de Radiodiagnóstico. España.

4. Centro de Diagnóstico y Resonancia Magnética de Salamanca. España.

5. Hospital Universitario de Salamanca. Servicio de Endocrinología Pediátrica. España.

\section{HYPOTHALAMIC HAMARTOMA ASSOCIATED WITH ARACH- NOID CYST: REPORT OF A CASE}

\begin{abstract}
Hypothalamic hamartomas are heterotopias of the nervous tissue which are similar to the grey matter of the hypothalamus. They may cause precocious puberty and/or gelastic seizures. We present the case of a patient who began her pubertal development at the age of 9 months. Cranial magnetic resonance imaging showed a hypothalamic hamartoma associated with middle cranial fossa and pineal region arachnoid cysts, a very rare association.
\end{abstract}

Keywords: Precocious puberty, gelastic seizures, hypothalamic hamartoma, arachnoid cyst, hypothalamic tumors.

Resumen: Los hamartomas hipotalámicos son heterotopias de tejido nervioso que se asemejan a la sustancia gris del hipotálamo. Pueden causar pubertad precoz y/o crisis gelásticas. Presentamos el caso de una paciente que a los 9 meses de edad comenzó desarrollo puberal y que en la resonancia magnética craneal se objetivó un hamartoma hipotalámico asociado a dos quistes aracnoideos, uno en fosa craneal media y otro en región pineal, asociación que es muy infrecuente.

Palabras clave: Pubertad precoz, crisis gelásticas, hamartoma hipotalámico, quiste aracnoideo, tumores hipotalámicos.

\section{Introducción}

El hamartoma hipotalámico $(\mathrm{HH})$ es una heterotopia congénita de tejido nervioso dependiente del hipotálamo. Es una lesión infrecuente que se

Martín D y cols. Hamartoma hipotalámico asociado a dos quistes aracnoideos. A propósito de un caso. Rev Chil Radiol 2008; 14: 6-8. ISSN 0717-9308.

Correspondencia: Dra. Diana Martín H. dianamh@gmail.com caracteriza clínicamente por el desarrollo de pubertad precoz y/o convulsiones gelásticas. Con el empleo de la resonancia magnética (RM) está aumentando su detección. Se ha sugerido una relación entre las características morfológicas de la lesión y el cuadro clínico asociado, que implica una importancia pronóstica y terapéutica. Presentamos un caso con dos quistes aracnoideos asociados en fosa craneal media derecha y región pineal.

\section{Caso clínico}

Niña de 9 meses de edad, que consulta por inicio de desarrollo de vello púbico y botón mamario. El estradiol basal se encuentra elevado y el test de estimulación con LH-RH muestra una respuesta elevada de LH y un cuociente elevado de LH/FSH. Estos datos permiten realizar el diagnóstico de pubertad precoz central, por lo que se le realiza una RM craneal en la que se objetiva una lesión supraselar pedunculada dependiente del tuber cinereum de 0,6 $\mathrm{cm}$ de diámetro, homogénea y bien delimitada, isointensa con la sustancia gris en todas las secuencias y que no se realza tras la administración de contraste (Figura 1). Además se objetivan dos lesiones extraaxiales localizadas en fosa craneal media derecha y en región pineal (Figura 2), isointensas respecto del líquido cefalorraquídeo en todas las secuencias y que no muestran restricción en las secuencias de difusión. En base a estos datos clínicos y morfológicos se realiza el diagnóstico de $\mathrm{HH}$ asociado a dos quistes aracnoideos. Se inicia tratamiento con Triptorelina, objetivándose una buena evolución clínica.

\section{Discusión}

Se define como pubertad precoz la aparición de los caracteres sexuales secundarios antes de los ocho años en las mujeres y de los nueve años en los varones.

La mayor parte de los casos de pubertad precoz de origen central son idiopáticos. Entre las causas de pubertad precoz central no idiopáticas se incluyen 

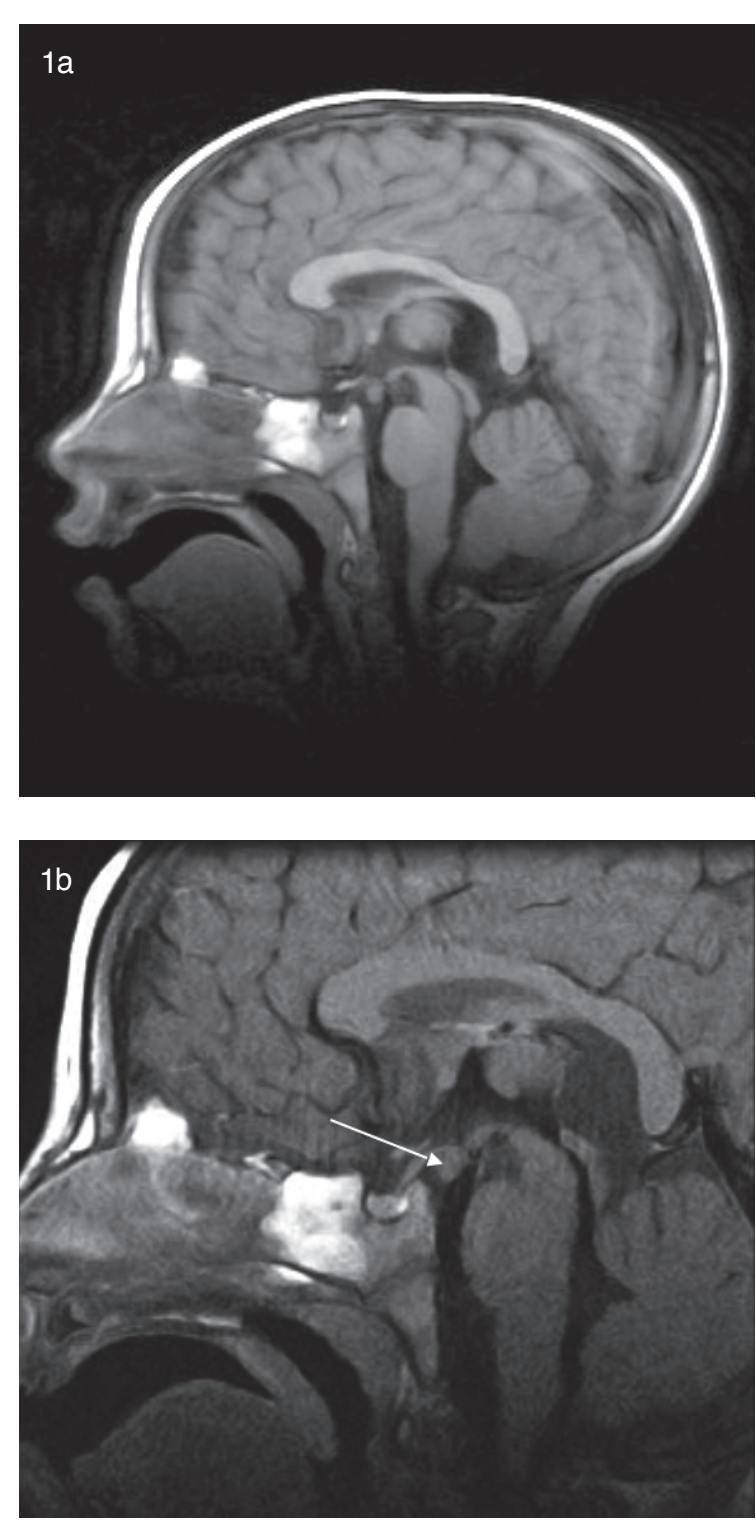

Figura 1. Imagen sagital potenciada en T1, previo (a) $y$ posterior (b) a la administración de contraste ev. Lesión nodular dependiente del tuber cinereum, isointensa respecto a la sustancia gris, que no muestra realce tras la administración de contraste, que corresponde a quiste aracnoideo en región pineal, que deforma discretamente el tectum mesencefálico.

las neoplasias primarias (astrocitoma, tumores de células germinales, craneofaringioma, ependimoma, adenoma hipofisario, pinealoma), alteraciones congénitas (hamartoma hipotalámico, quistes aracnoideos supraselares, facomatosis, displasia septo-óptica) y enfermedades adquiridas, como lesiones inflamatorias, iatrogénicas y traumáticas ${ }^{(1-3)}$.

El HH es una heterotopia congénita de sustancia gris que consiste en neuronas maduras y células gliales, entre las que se disponen fibras mielinizadas y sin mielinizar. La detección de $\mathrm{HH}$ ha aumentado con el empleo de la RM.
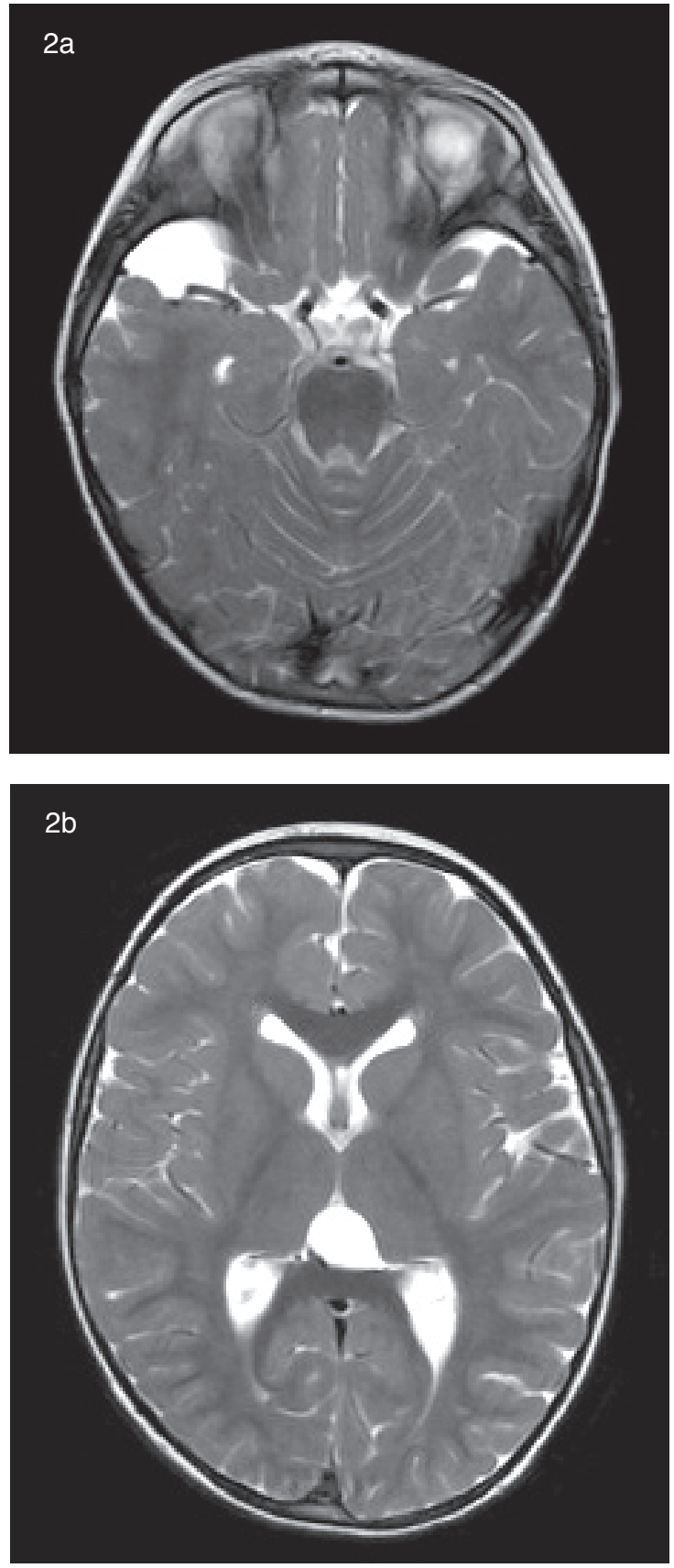

Figura 2. Imagen axial potenciada en T2: Quiste aracnoideo en fosa craneal media derecha (a) y región pineal (b).

Aparece con la misma frecuencia en varones y en mujeres y es causa de PPC a edades tempranas (0-3 años). Presenta células secretoras de GnRH o células astrogliales secretoras de TGF-alfa (transforming growth factor alpha), que está implicado en la liberación pulsátil de $\mathrm{LH}-\mathrm{RH}^{(4)}$. Otra teoría defiende que la compresión mecánica del hipotálamo impide que este ejerza su acción inhibitoria normal sobre las células gonadotropas de la hipófisis ${ }^{(1-3)}$. Los $\mathrm{HH}$ pueden producir convulsiones gelásticas resistentes al tratamiento que conllevan disfunción cognitiva y problemas de comportamiento. 
Excepcionalmente se puede manifestar con acromegalia, síndrome diencefálico o deterioro visual progresivo o formando parte de síndrome de PallisterHall. También puede coexistir con otras anomalías intracraneales como agenesia del cuerpo calloso e hipoplasia cerebelosa.

La asociación de quistes aracnoideos y $\mathrm{HH}$ es infrecuente, existiendo pocos casos descritos en la literatura, tanto en región supraselar como en fosa craneal media ${ }^{(5-7)}$. Las etiologías del quiste aracnoideo son múltiples. La mayor parte son lesiones congénitas que se producen como consecuencia de una alteración en el desarrollo de la aracnoides, entre la semana 14 y la 17 de gestación. También pueden ser adquiridos como consecuencia de una inflamación del espacio subaracnoideo por leptomeningitis, traumatismos, hemorragia subaracnoidea o estar asociados a tumores extraaxiales o intraaxiales que contacten con el espacio subaracnoideo. La patogenia de los quistes aracnoideos congénitos asociados a hamartoma hipotalámico no se conoce con exactitud, pero hay autores que sugieren que las proyecciones de tejido hipotalámico ectópico en el espacio subaracnoideo perimesencefálico puede alterar el desarrollo del mismo y conducir a la formación de quistes aracnoideos ${ }^{(5)}$.

El HH aparece como un nódulo sólido homogéneo, generalmente de pequeño tamaño y bien delimitado, que se proyecta desde el tuber cinereum y/o cuerpos mamilares inferiormente ocupando la cisterna supraselar. Su intensidad de señal es similar a la sustancia gris en secuencias potenciadas en T1 (Figura 3) y similar o algo elevada en aquellas potenciadas en T2 y no se realza tras la administración de contraste; no suelen calcificar ni sufrir hemorragias ${ }^{(4)}$.

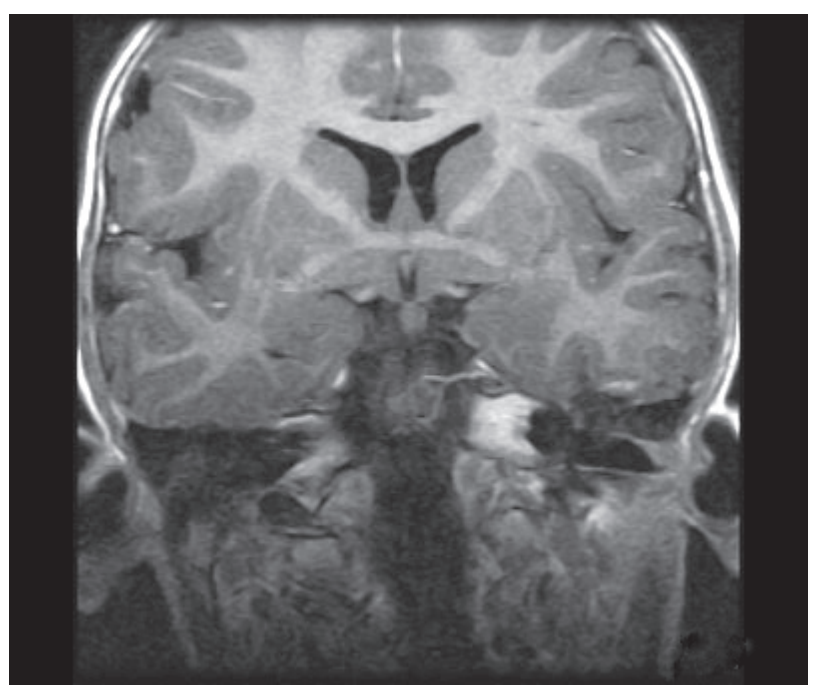

Figura 3. Imagen coronal potenciada en T1: Hamartoma hipotalámico.
Se ha sugerido una asociación entre las características imaginológicas del $\mathrm{HH}$ y la clínica que produce: las lesiones pedunculadas y de situación parahipotalámica suelen asociarse a pubertad precoz mientras que las lesiones grandes (mayores de $10 \mathrm{~mm}$ de diámetro), sésiles y de crecimiento intrahipotalámico se asocian a convulsiones gelásticas y/o complejas ${ }^{(4,8)}$.

El diagnóstico diferencial de tumor hipotalámico en niños que padecen pubertad precoz incluye además: astrocitoma, glioma óptico, tumor de células germinales y craneofaringioma. Todas estas lesiones suelen presentar captación tras la administración de contraste y además la presencia de calcificaciones es frecuente en el craniofaringioma.

El tratamiento de los $\mathrm{HH}$ productores de PPC consiste en la administración de agonistas de $\mathrm{GnRH}$, que frenan la maduración puberal y facilitan la regresión de los caracteres sexuales secundarios. EI tratamiento quirúrgico se reserva para los raros casos en los que falla el tratamiento médico y representa una alternativa importante a los fármacos antiepilépticos en los $\mathrm{HH}$ productores de síntomas epilépticos, ya que éstos generalmente no son suficientes para el control de las crisis ${ }^{(4)}$.

En el seguimiento del $\mathrm{HH}$ se recomienda un estudio tardío de control mediante RM para demostrar la falta de crecimiento y la estabilidad en la forma de la lesión y en su intensidad de señal.

\section{Bibliografía}

1. Partsch C.J, Sippell W.G. Pathogenesis and epidemilogy of precocious puberty. Effects of exogenous oestrogens. Human reproduction Update. 2001; 7(3): 292-302.

2. Trivin C, Couto-Silva AC, Sainte-Rose C, Chemaitilly W, Califa C, Doz F, et al. Presentation and evolution of organic central precocious puberty according to the type of CNS lesion. Clin Endocrinol (Oxf) . 2006; 65(2): 239-245.

3. Rivarola, Belgorosky A, Mendilaharzu H, Vidal G. Precocious puberty in children with tumours of the suprasellar and pineal areas: organic central precocious puberty. Acta Paediatr. 2001; 90(7): 751-756.

4. Arita K, Kurisu K, Kiura Y, lida K, Otsubo H. Hypothalamic hamartoma. Neurol Med Chir. 2005; 45, 221-231.

5. Nishio S, Morioka T, Hamada Y, Kuromaru R, Fukui M. Hypothalamic hamartoma associated with an arachnoid cyst. J Clin Neuroscience. 2001; 8(1); 46-47.

6. Goda M, Tashima A, Isono M, Hori S, Kimba Y. A case of hypothalamic hamartoma associated with an aracnoid cyst. Neurol Med Chir (Tokyo).1999; 15(9): 490-2.

7. Booth T, Timmons C, Shapiro K, Rollins N. Pre- and postnatal MR imaging of hypothalamic hamartomas associated with arachnoid cysts. Am J Neuroradiol. 2004; 25: 1.283-1.285.

8. Jung H, Neumaier Probst E, Hauffa B.P, Partsch C-J, Dammann O. Association of morphological characteristics with precocious puberty and/or gelastic seizures in hypothalamic hamartoma. J Clin Endocrinol Metab. 2003; 88(10): 4.590-4.595. 